\title{
Establishment of Minimally Invasive Thoracic Surgery Program
}

\author{
Jong Ho Cho, M.D., Ph.D. \\ Department of Thoracic and Cardiovascular Surgery, Samsung Medical Center, Sungkyunkwan University School of Medicine, Seoul, Korea
}

\author{
ARTICLE INFO \\ Received June 10, 2021 \\ Accepted July 16, 2021 \\ Corresponding author \\ Jong Ho Cho \\ Tel 82-2-3410-1696 \\ Fax 82-2-3410-1696 \\ E-mail jongho.cho@gmail.com \\ ORCID \\ https://orcid.org/0000-0003-3362-4621
}

\begin{abstract}
The establishment of minimally invasive surgery is a complex and difficult task. Video-assisted thoracic surgery (VATS) refers to a minimally invasive surgical technique that represents a less invasive approach to thoracic surgery using thoracoscopy. For lung cancer or esophageal cancer surgery, planning and establishing a team for minimally invasive surgery for the first time is not a simple task. Technical advances in surgical devices and the enhanced skill of surgeons are cornerstones of the development of minimally invasive surgery. Here, we review the meaning of minimally invasive thoracic surgery and discuss how to establish a team approach for VATS procedures.
\end{abstract}

Keywords: Video-assisted thoracic surgery, Minimally invasive surgery, Surgery

\section{Introduction}

In recent years, there has been a shift towards video-assisted thoracic surgery (VATS) in the field of thoracic surgery. VATS refers to surgery to remove lung cancer, esophageal cancer, or mediastinal tumors without rib cutting or sternotomy, as was done in the past. In general, minimally invasive surgery is associated with less pain, shorter hospital stays, and fewer complications [1]. It also has allowed surgeons to perform new, complex, and conventional procedures without the major risks associated with a large open incision.

VATS is just one of the first types of minimally invasive surgery. One subtype of minimally invasive surgery is robotic surgery, which offers surgeons a magnified, 3-dimensional view of the surgical site with better flexibility and control. We can use these advanced robotic surgical techniques to achieve excellent surgical outcomes and safety in most cases of thoracic surgery.

The technical and oncological safety of thoracoscopic surgery for early-stage lung cancer is considered acceptable worldwide $[2,3]$. It has recently been reported that the majority of thoracic surgical procedures can be performed with minimal invasive surgery due to advances in the development of surgical techniques, even in complex cases of patients with lung cancer [4]. However, even with minimally invasive surgery, there are risks of complications in- volving anesthesia, major bleeding, and infection [5]. Therefore, it is important to prepare for serious complications, such as massive bleeding from the pulmonary artery during VATS, with a backup plan always in mind [6].

\section{How to set up an excellent surgical team for minimally invasive surgery}

\section{Team approach}

It is unusual for patients to receive more attention from the medical team than during surgery. Patients are prepared by 1 surgeon, 1 or 2 assistants, anesthesiologists, and nursing staff. Each member of the surgical team has his or her own function, but the surgical team is like playing a symphony, so each team member must collaborate with his or her colleagues to achieve good results. The patient's life is in the hands of the operating team, so there can be no such thing as properness for its own sake in the operating room. To bring the best results for the patient, everyone must work quickly and effectively, often in desperate situations. They need to be familiar with surgical procedures, how to operate machines (e.g., surgical robot systems), instruments, and surgical items, understand each other, trust each other, cooperate, and be considerate. A person who does not devote his or her whole heart to working as a good member of the team, and who instead tries to work at 
his own level, creates dissonance in teamwork, making it difficult to work together in the operating room.

A surgical team includes an array of specialists who need to work in close cooperation for the operation to succeed. A single error, miscommunication, or slow response can have disastrous consequences. To develop the best surgical team, we have to be the best possible leader. An excellent article on the approach to building a surgical team approach was published in the Harvard Business Review [7]. They presented several factors for the development of a successful team, as described below:

\section{Creating a learning team}

The surgeon as a team leader should follow the up-todate knowledge in secondary medical textbooks, quickly acquire new medical skills, share them with fellow doctors, and build a system while maintaining the 4 components of teamwork [7]. The new paradigm of health care focuses increasingly on interdisciplinary teams as its core unit. The increasing complexity of the surgical environment has changed the delivery of surgical care and has increasingly led to the formation of multidisciplinary provider teams replacing the more traditional individualistic surgeon-assistant-nurse relationship. The challenges are considerable, even if we are already faced with tight competition, especially in the 80-hour working-week environment of surgical resident programs. It may be a good strategy for surgical educators to collaborate with other colleagues who have conducted training for a certain period.

\section{Framing the challenge}

Properly framing the challenge is critical to our success in the field of surgery. A frame challenge is where someone answers a question in a wholly different way that the asker never expected, but in a way that he or she feels will solve the problem. The difficulty of introducing new surgical procedures places more stress on team members than usual for minimally invasive surgery. Surgeons must help their team embrace and internalize the legitimacy of this important new challenge regarding new surgical techniques. Even so, identifying the right challenge for innovation is particularly crucial for the surgical team.

\section{Creating an environment of psychological safety that fosters communication and innovation}

It is important to have an environment where the leader's innovation and creativity are properly accepted, and team members can exchange feedback with each other. A belief that one will not be punished or humiliated for speaking up with ideas, questions, or mistakes is important for both a leader and team members. This relates to assistants feeling empowered to express an idea or contribution fully, without fear of negative consequences to themselves or their status [7]. It includes being courageous enough to own one's mistakes and turn them into learning, and to trust that one's work environment and co-workers will not shame one for doing so [7].

\section{Preparation}

It is important to always prepare the basic rules of surgery, the instruments used, the order of surgery, and the materials for surgery. To pack all the items to be prepared before surgery, possible surgical materials and instruments should be listed and prepared.

Surgical practice has evolved rapidly, driven in part by the increasing complexity of patients, the severity of their diseases, rapidly evolving new technologies, and a deluge of evidence to support best practices. For example, the application of robotic surgical systems for lobectomy was first described in 2002 [8]. Since then, robotic-assisted VATS lobectomy has become more popular for the treatment of lung cancer, and the rapid evolution of technology has prompted many centers to initiate robotic surgical procedures $[9,10]$.

\section{Prepare for the worst, hope for the best}

"Great deeds are usually wrought at great risks" - Herodotus (484-402 B.C.)

\section{Conversion to thoracotomy or sternotomy}

If one has to switch to thoracotomy or sternotomy from VATS, one should not hesitate. Surgeons must always be ready for the possibility of open conversion and should rehearse this possibility with team members. For VATS lobectomy, the incidence of intraoperative conversion to an open approach ranges from $5 \%$ to $23 \%$, and nearly half the conversions are performed emergently $[11,12]$. In fact, the surgeon should set his or her appropriate criteria for conversion to open thoracotomy or sternotomy. For example, Dunning and Walker [13] suggested that a surgical team should use a reasonable parameter for the timing of conversion, which may be if the pulmonary artery has not been divided within 1.5-2 hours.

\section{Management of intraoperative bleeding}

Although an increasing number of studies have found 
that VATS lobectomy appears to be superior to conventional open lobectomy for perioperative outcomes $[1,14]$, even minimally invasive surgery still involves risks for intraoperative complications such as intraoperative bleeding. A major concern in VATS procedures is that trying to dissect a pulmonary vessel or another large vessel during the surgery may lead to severe bleeding, which is troublesome to control with small ports. Surgeons should keep a sponge stick available to immediately apply pressure to control bleeding when it happens. Here are the author's own safety rules to manage massive bleeding during VATS lobectomy:

Management when massive bleeding occurs during VATS lobectomy

(1) Ask for help: anesthesiologist, cardiac surgeon, or senior thoracic surgeon.

(2) Blood transfusion: ask for a massive transfusion from nursing staff or others.

(3) Calm down and compress: control your own heartbeat, prepare a sponge stick, and immediately apply pressure with it.

(4) Decide whether thoracotomy is really needed.

(5) Expose the main pulmonary trunk or proximal part of the injured vessel.

(6) Fix the injured vessel with a suture or fibrin sealant patch.

Check possible clues to prevent reoperation before leaving the operating room after surgery.

Before leaving the operating room, it is necessary to make sure that the entire surgical procedure has been performed well. Especially before closing after lung cancer surgery, it is necessary to check several factors to prevent postoperative complications such as bronchopleural fistula, torsion, bleeding, and prolonged air leak. Below is a list of the items that the author always must verify before leaving the operating room after lobectomy.

Checklist before leaving the operating room after lobectomy

(1) Airway: check the bronchial stump.

(2) Bleeding: check the vascular stump (pulmonary artery/vein, bronchial artery) and incision site.

(3) Circulation: check venous drainage.

(4) Drain: check the appropriate position of the chest tube or catheter.

(5) Expansion: check that there is enough pulmonary expansion.

(6) Fissure: check for a complete fissure (possible torsion).

\section{Record the quality notes and feedback}

Furthermore, surgeons must keep meticulous perioperative notes and record a quality report, which is mandatory for all new surgical procedures. Defining and measuring how each of a variety of factors affects outcomes is an important but challenging objective. As for perioperative complications, the procedure and process during which they occurred, how the results appeared, and the resulting solutions should be recorded in detail so that the procedure can develop as experience is accumulated. Based on these records, more complex surgical procedures can be performed using minimally invasive surgery, as is happening in the field at this moment.

\section{Conclusion}

Surgeons should constantly think about why we do minimally invasive surgery and look at it from the patient's point of view. Of course, it is acceptable for any surgeon to pursue minimally invasive surgery because it can provide the patient with better surgical outcomes, such as a quicker recovery and fewer complications. However, surgeons may try to avoid minimally invasive surgery in complicated and difficult cases. Minimally invasive surgery requires some risk and preparation to be successful. We must constantly work with our team members to continue developing our surgical skills, easily adapt to new equipment, and use new techniques freely.

Lastly, I would like to share with you the saying by Professor Young Mog Shim, my teacher and mentor:

"Surgery should be done using the best surgical method for the patient, not a comfortable and easy surgery for the surgeon."

\section{Conflict of interest}

No potential conflict of interest relevant to this article was reported.

\section{ORCID}

Jong Ho Cho: https://orcid.org/0000-0003-3362-4621

\section{References}

1. Nwogu CE, D’Cunha J, Pang H, et al. VATS lobectomy has better perioperative outcomes than open lobectomy: CALGB 31001, an ancillary analysis of CALGB 140202 (Alliance). Ann Thorac Surg 
2015;99:399-405.

2. Swanson SJ, Herndon JE 2nd, D'Amico TA, et al. Video-assisted thoracic surgery lobectomy: report of CALGB 39802: a prospective, multi-institution feasibility study. J Clin Oncol 2007;25:4993-7.

3. Yan TD, Black D, Bannon PG, McCaughan BC. Systematic review and meta-analysis of randomized and nonrandomized trials on safety and efficacy of video-assisted thoracic surgery lobectomy for early-stage non-small-cell lung cancer. J Clin Oncol 2009;27:2553-62.

4. Solli P, Bertolaccini L, Droghetti A, et al. 2016 Annual report from the Italian VATS Group. Future Oncol 2018;14(6s):23-8.

5. Boffa DJ, Kosinski AS, Furnary AP, et al. Minimally invasive lung cancer surgery performed by thoracic surgeons as effective as thoracotomy. J Clin Oncol 2018;36:2378-85.

6. Liang C, Wen H, Guo Y, et al. Severe intraoperative complications during VATS lobectomy compared with thoracotomy lobectomy for early stage non-small cell lung cancer. J Thorac Dis 2013;5:513-7.

7. Edmondson A, Bohmer R, Pisano G. Speeding up team learning. Harv Bus Rev 2001;79:125-32.

8. Melfi FM, Menconi GF, Mariani AM, Angeletti CA. Early experience with robotic technology for thoracoscopic surgery. Eur J Cardiothorac Surg 2002;21:864-8.
9. Moller T, Egberts JH, Eichhorn M, et al. Current status and evolution of robotic-assisted thoracic surgery in Germany: results from a nationwide survey. J Thorac Dis 2019;11:4807-15.

10. Zirafa CC, Romano G, Key TH, Davini F, Melfi F. The evolution of robotic thoracic surgery. Ann Cardiothorac Surg 2019;8:210-7.

11. Lim CG, Shin KM, Lim JS, et al. Predictors of conversion to thoracotomy during video-assisted thoracoscopic surgery lobectomy in lung cancer: additional predictive value of FDG-PET/CT in a tuberculosis endemic region. J Thorac Dis 2017;9:2427-36.

12. Samson P, Guitron J, Reed MF, Hanseman DJ, Starnes SL. Predictors of conversion to thoracotomy for video-assisted thoracoscopic lobectomy: a retrospective analysis and the influence of computed tomography-based calcification assessment. J Thorac Cardiovasc Surg 2013;145:1512-8.

13. Dunning J, Walker WS. How to set up a VATS lobectomy program. Ann Cardiothorac Surg 2012;1:43-6.

14. Puri V, Patel A, Majumder K, et al. Intraoperative conversion from video-assisted thoracoscopic surgery lobectomy to open thoracotomy: a study of causes and implications. J Thorac Cardiovasc Surg 2015;149:55-62. 